\title{
Intervención cognitivo conductual para mujeres con trastorno depresivo persistente: sintomatología ansiosa, depresiva y satisfacción vital
}

\section{Cognitive behavioural intervention for women with persistent depressive disorder: anxiety and depression symptoms and life satisfaction}

\author{
Laura Lacomba-Trejo ${ }^{1}$, Isabel María García-Cuenca² y José Manuel Sanz-Cruces ${ }^{3}$ \\ ${ }^{1}$ Universidad de Valencia. Valencia, España \\ ${ }^{2}$ Consorcio Hospital General Universitario de Valencia, España \\ ${ }^{3}$ Hospital Universitari Institut Pere Mata, España
}

\begin{abstract}
Resumen: Antecedentes. El trastorno depresivo persistente (TDP) es una afectación del estado de ánimo frecuente en mujeres. Se ha empleado la terapia cognitivo conductual (TCC), la terapia cognitiva basada en mindfulness y la terapia centrada en la compasión de forma aislada. Objetivos. Evaluar la eficacia de un programa de intervención grupal multicomponente. Método. Tras la firma del consentimiento informado, las participantes $(n=6)$ respondieron (pre, post intervención y seguimiento) cuestionarios sobre: ansiedad, depresión, satisfacción con la vida y satisfacción con la intervención. Resultados. Nuestros resultados previos al tratamiento muestran niveles elevados de sintomatología ansiosa y depresiva y baja satisfacción vital, que mejoran tras la intervención y se mantienen estables en el seguimiento a los seis meses. Conclusiones. Por ello, concluimos que
\end{abstract}

\footnotetext{
Para citar este artículo: Lacomba-Terjo, L., García-Cuenca, I. M. y Sanz-Cruces, J. M. (2021). Intervención cognitivo conductual para mujeres con trastorno depresivo persistente: sintomatología ansiosa, depresiva y satisfacción vital. Revista Clínica Contemporánea, 12(1), Artículo e3. https://doi. org/10.5093/cc2021a1

Laura Lacomba-Trejo es Personal Docente e Investigador en Formación. Departamento de Personalidad, Evaluación y Tratamientos Psicológicos . Universidad de Valencia. Valencia, España.

Isabel María-Cuenca es Psicóloga Clínica. Unidad de Psicología Clínica y de la Salud. Consorcio Hospital General Universitario de Valencia. Valencia, España.

José Manuel Sanz-Cruces es Psicólogo Clínico. Hospital Universitari Institut Pere Mata. Reus. Tarragona, España.
}

La correspondencia sobre este artículo debe enviarse a Isabel María García-Cuenca, Consorcio Hospital General Universitario de Valencia, Unidad de Psicología Clínica y de la Salud, Avenida Tres Cruces, 2, 46014, Valencia (España).E-mail: isabelgarciapsicologiaclinica@gmail.com

(cc) BY-NC-ND Este es un artículo Open Access bajo la licencia CC BY-NC-ND. 
el tratamiento multicomponente puede mejorar la salud emocional de las pacientes con TDP, disminuyendo su sintomatología ansiosa y depresiva y aumentando su satisfacción con la vida.

Palabras clave: Trastorno depresivo persistente, terapia cognitivo conductual, perspectiva de género, satisfacción vital

\begin{abstract}
Background. Persistent depressive disorder (PDD) is a common mood disorder in women. Cognitive behavioral therapy (CBT), mindfulness-based cognitive therapy, and compassion-focused therapy have been used separately. Objectives. To assess the efficacy of a multicomponent group intervention program. Method. After signing an informed consent, participants $(n=6)$ answered (before and after intervention and follow-up) questionnaires on anxiety, depression, life satisfaction, and satisfaction with the intervention. Results. Our pre-treatment results showed high levels of anxious and depressive symptoms and low life satisfaction, which improved after the intervention and remained stable at the six-month follow-up. Conclusions. It is therefore concluded that multicomponent treatment can improve the emotional health of patients with PDD, decreasing their anxious and depressive symptoms and increasing their satisfaction with life.

Keywords: Persistent depressive disorder, cognitive behavioral therapy, gender perspective, life satisfaction
\end{abstract}

Según datos de la OMS (2017), en 2015 en España se alcanzaban casi los 3.000 .000 de personas (5.2\%) con depresión, siendo uno de los países europeos donde más personas la padecen, viéndose como aumentan las cifras de forma vertiginosa cada año, y siendo la principal causa de discapacidad a nivel mundial. El curso de los trastornos depresivos suele ser crónico (Institute for Health Metrics and Evaluation [IHME], 2017), por lo que puede tener grandes repercusiones personales, sanitarias y sociales (Rossel et al., 2020). Por ello, su tratamiento debe tener en cuenta: la psicoterapia, los elementos psicoeducativos y familiares y el tratamiento psicofarmacológico (Nobile et al., 2003). No obstante, aunque existen tratamientos eficaces para los trastornos depresivos, más de la mitad de las personas que los necesitan no los reciben (OMS, 2018).

Se ha observado que los trastornos del estado del ánimo suelen estar presente durante toda la vida, observándose síntomas de forma recurrente y fluctuante (Barnhofer, 2019), aspecto que es todavía más reseñable en el caso del trastorno depresivo persistente (TDP). El TDP, en el DSM-IV-TR denominado distimia, es un trastorno del estado de ánimo que se caracteriza por la presencia de la siguiente sintomatología durante dos años o más: bajo estado de ánimo crónico, fatiga, falta de concentración, sentimientos de desesperanza, insatisfacción y baja autoestima. Pudiendo aparecer pérdida o aumento del sueño y del peso, generando una gran interferencia en la vida diaria de quienes lo padecen (Asociación Americana de Psiquiatría [APA], 2014). A pesar de ser una categoría diferenciada de la depresión mayor, existen grandes similitudes, pues los factores de riesgo y protección son similares, ambos trastornos responden de forma favorable a mismos tratamientos y en estudios longitudinales, se observa a menudo la migración entre categorías (Uher, 2014). Entre los principales factores de riesgo en el desarrollo y mantenimiento del TDP, se encuentra el afrontamiento centrado en la emoción (Gallardo-Moreno et al., 2013) así como la disminución de la imagen corporal, el deterioro del funcionamiento físico, los cambios en la perspectiva de vida, la dependencia, los estigmas sociales asociados (Almada et al., 2014) y la disminución de la satisfacción vital (Acosta, 2019; Teixeira y Prebianchi, 2019).

El TDP está presente aproximadamente en un 3\% de la población, y como otros trastornos emocionales, es mucho más frecuente en mujeres (5.25\%) que en hombres (1.85\%) (de Francisco y Blasco, 2015; Ministerio de Sanidad y Consumo, 2007). Posiblemente por los factores de vulnerabilidad psicosociales, como: la desigualdad de oportunidades, la discriminación en el ámbito social, la mayor exposición a situaciones 
vitales estresantes, especialmente agresiones, el cuidado de personas dependientes o la presión social (Montesó-Curto, 2014; Pla et al., 2013; Cortés, Lacomba-Trejo y Pérez-Marín, 2020). Por ello, entre los factores de riesgo que predicen un peor curso y evolución de los trastornos del estado de ánimo se encuentra el hecho de ser mujer. Además de lo anterior, factores como: vivir en un país con mayores ingresos, tener menor nivel de escolaridad, tener una edad avanzada, baja autoestima, pobres habilidades sociales y poco apoyo social, y presentar comorbilidad con otras dificultades emocionales (otros trastornos del estado de ánimo, trastornos ansiosos, trastornos por consumo de sustancias y trastornos de personalidad) (Eguskiza, 2012), se asocian con un peor curso y resultado del trastorno (Guhn et al., 2019; Gureje, 2011; Nobile et al., 2003; Saenz-Miguel et al., 2019; Torres et al., 2019).

Pese a su gran prevalencia, poco se sabe hasta la fecha del TDP (Guhn et al., 2019). A diferencia de las personas que padecen un único episodio depresivo, las personas que sufren TDP, tienen mayor probabilidad de padecer otros trastornos psicológicos (Angst et al., 2009; Eguskiza, 2012) y de intentar múltiples tratamientos sin éxito (Cuijpers et al., 2011). En este sentido, se ha evidenciado que el $40 \%$ de las personas con TDP que se someten a tratamiento farmacológico, no responden al mismo (Ebrahimi et al., 2013). Del mismo modo, cuando los pacientes tienen varios episodios depresivos el riesgo de que se repitan es de un 90\% y, en estas situaciones la TCC sola o la terapia interpersonal, se han asociado con mayores índices de recaídas (Korman y Caray, 2012). Ahora bien, cuando estos pacientes no reciben tratamiento las probabilidades de remisión del TDP son del 10\% y obviamente mejoran si se tiene tratamiento (Ministerio de Salud, 2013). No obstante, en cuanto a la intervención en TDP existe un volumen mucho menor de investigaciones, siendo la TCC la que se ha introducido como una de las terapias más eficaces (Narbona, Gómez y Martínez, 1993; Berrío, 2019). Se ha observado que, en este colectivo, la respuesta ante el tratamiento es menor, por lo que son necesarios tratamientos novedosos para atender a esta población, que se ve gravemente afectada (Cujipers et al., 2017). Es por ello, que se hace necesario generar nuevas tendencias de tratamiento, teniendo en cuenta que la terapia cognitivo conductual (TCC) es la terapia recomendada para los trastornos depresivos en general (Feldman, 2007; Ministerio de Sanidad, Servicios Sociales e Igualdad, 2014; Pérez y García, 2001). A pesar de ello, su eficacia para tratar este diagnóstico es todavía desconocida (Ebrahimi et al., 2013) pues generalmente los estudios consideran a personas con diferentes trastornos del estado de ánimo, y no dirigen las intervenciones exclusivamente para personas con TDP (Cruijpers et al., 2008; Pinquart et al., 2006; Rosenbaum et al., 2014).

Estos resultados han provocado una búsqueda para mejorar la eficacia en el tratamiento del TDP. En este sentido, se ha propuesto que se incluyan elementos para mejorar las habilidades sociales y emocionales dentro del tratamiento (Guhn et al., 2019). Otras propuestas señalan la inclusión de la perspectiva de género, de elementos de la terapia cognitiva basada en mindfulness (MBCT) y la terapia centrada en la compasión (Alonso-Llácer, 2017; Barnhofer, 2019; Cerdá et al., 2017; Hamidian et al., 2013; Moscoso, 2019; Muñoz et al., 2008), es decir, incluir los enfoques de tercera ola de la TCC. La tercera ola muestra una orientación más experiencial y tiene en cuenta que las estrategias de modificación conductual son indirectas. Desde esta perspectiva se intenta: disminuir el control, aceptar las emociones, sensaciones y vivencias (Vallejo, 2006). De este modo, se puede promover procesos de cambios que puedan ser sostenibles y que ayuden a la persona en tratamiento a aceptar y procesar el estado de ánimo y los pensamientos asociados (Barnhofer, 2019). Todas estas recomendaciones se han mostrado útiles siendo el formato grupal el de elección (Latorre et al., 2012; Sanz-Cruces et al., 2016; Sanz-Cruces et al., 2018). Ya que el trabajo grupal puede aumentar el apoyo social, aspecto que a menudo se ve mermado en estos pacientes. El apoyo social puede funcionar como amortiguador del dolor, disminuyendo así los síntomas ansiosos y depresivos y aumentando la satisfacción vital (Acosta, 2019; Teixeira y Prebianchi, 2019). Por ello, el objetivo general de nuestra investigación fue evaluar la eficacia un tratamiento multicomponente realizado en un contexto clínico dirigido a personas con TDP. Este tratamiento se realizará en una muestra piloto que sirviera de experiencia para futuras intervenciones grupales, buscando disminuir los síntomas ansioso-depresivos y aumentar el nivel de satisfacción vital en mujeres con TDP. Se espera (H1) que tras el tratamiento disminuyan los síntomas ansiosos y depresivos y aumente la satisfacción vital. 


\section{Metodología}

\section{Participantes}

Se recibieron 13 derivaciones de la unidad de salud mental, de las cuales nueve participaron en las sesiones de psicoterapia, tres de ellas abandonaron el tratamiento y finalmente seis mujeres participaron en la investigación. El diagrama de evolución de la muestra se puede consultar en la Figura 1. Las participantes $(\mathrm{n}=6)$ tenían entre 43 y 61 años $(M=52.50, D T=6.95)$, siendo todas ellas mujeres. Así un $66.70 \%$ tomaba tratamiento farmacológico que consistía en un ansiolítico y un antidepresivo. De media recibían el tratamiento farmacológico desde hace casi tres años $(M=33.35$; $\mathrm{DT}=18.06)(\mathrm{Min}=24$; Máx $=60)$. Asimismo, estaban en tratamiento psicológico individual de forma coadyuvante desde hace 4 a 55 meses $(\mathrm{M}=25.33$; $\mathrm{DT}=23.14)$.

Todas vivían acompañadas, estaban casadas o tenían pareja y tenían hijos. Así, un $66.70 \%$ tenía dos hijos, el 33.30\% restantes tenía 3 hijos. En cuanto a su situación laboral: el 33.30\% estaba desempleada, el 33.30\% con incapacidad temporal y el $33.30 \%$ se dedicaba a las tareas del hogar. En relación con su nivel de estudios: un $25 \%$ tenía estudios primarios incompletos, un $50 \%$ estudios primarios completos y un $25 \%$ estudios superiores. Como criterios de inclusión tuvimos en cuenta: a) ser mujer, b) mayor de edad, c) cuyo diagnóstico principal fuera TDP (mediante los criterios del DSM-5) y d) que estuviera interesada en participar en el tratamiento. Como criterios de exclusión se contemplaron: a) presentar trastornos de personalidad, psicóticos o por consumo de sustancias y b) mostrar ideas autolíticas activas. Estos aspectos se evaluaron consultando el historial médico de las participantes y realizándoles una entrevista clínica semiestructurada.

Figura 1. Evolución de la muestra

\section{SUPERVIVENCIA MUESTRAL}

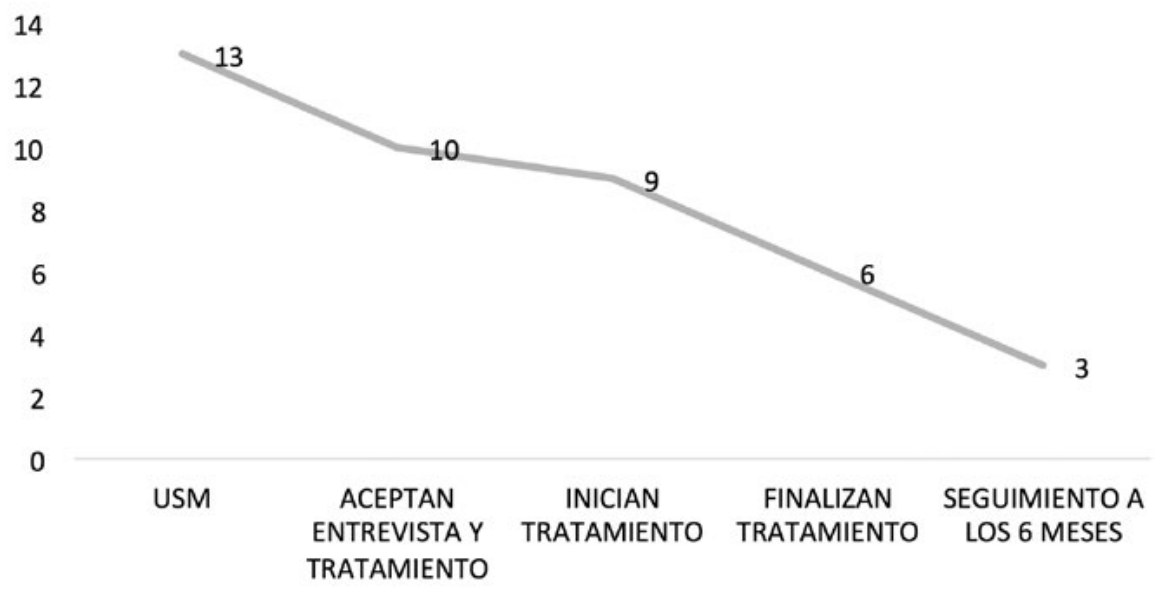

\section{Instrumentos}

Se utilizaron registros ad hoc para recoger información sobre las variables sociodemográficas de las participantes. Las variables psicológicas analizadas fueron:

- Inventario de Ansiedad Estado-Rasgo (STAI) (Spielberger et al., 1982). Es un autoinforme que consta de 20 ítems relacionados con la ansiedad-rasgo y otros 20 relacionados con la ansiedad-estado. El rango de las puntuaciones es de 0 a 60 en cada escala. En muestras clínicas se encuentran puntuaciones medias de 35.07 $(D T=11.07)$ para estado y de $35.67(D T=12.90)$ en rasgo. La fiabilidad test-retest es de 0.81 en la escala de ansiedad-rasgo, bastante más alta, como es lógico, que en la de ansiedad-estado $(r=0.40)$. La consistencia interna oscila de 0.86 a 0.93 en muestra clínica. Siendo un instrumento adecuado para diferenciar 
entre muestras con trastornos psicológicos y sin ellos (Ortuño-Sierra, García-Velasco, Inchausti, Debbané y Fonseca-Pedrero, 2016).

- Inventario de Depresión de Beck II (BDI-II) (Sanz et al., 2003). Autoinforme de 21 ítems que evalúa la intensidad de los síntomas depresivos de 0 a 63, considerándose puntuaciones superiores a 18 como sintomatología depresiva de interés (Steer y Beck, 1988). Se consideran valores menores o iguales a 9 (la media de la población general española), como criterios de recuperación clínicamente significativa de una depresión. El BDI-II ha mostrado adecuadas propiedades psicométricas en cuanto a la consistencia interna (0.87) y a la validez (Sanz et al., 2003).

- Escala de Satisfacción Vital (SWLS) (Diener et al., 1985; adaptación al español de Vázquez et al., 2013). Está compuesta por cinco ítems tipo Likert con 7 opciones de respuesta $(1=$ totalmente desacuerdo; $7=$ totalmente de acuerdo). Ha obtenido adecuada consistencia interna (0.84) (Atienza et al., 2000).

Tras el tratamiento, como medida de mejoría clínica se utilizó por una parte el alta de los pacientes de la Unidad de Salud Mental (USM), y por otra, una breve encuesta donde se les preguntaba por su percepción de mejoría respecto a la situación inicial. Para ello, las pacientes respondieron a tres afirmaciones de 1 (muy en desacuerdo) a 5 (muy de acuerdo), sobre: a) su experiencia subjetiva: "me siento mejor conmigo misma ahora que cuando empecé la terapia (ejemplo: con más autoestima, menos culpa, menos soledad)”, b) percepción de cambios conductuales: "la terapia me ha ayudado a hacer cambios en la forma de llevar mi vida, como tomar decisiones sobre mi vida y llevarlas a cabo (ejemplo: socializarme o iniciar actividades)" y c) alivio de los síntomas: "pienso que la terapia me ha ayudado a tener menos síntomas de ansiedad y depresión (ejemplo: menos dolores corporales, insomnio, pérdida de apetito, llanto o preocupación)".

Además de lo anterior, se administró de forma anónima una encuesta para conocer la satisfacción de las pacientes con el tratamiento grupal. Esta encuesta consistió en 25 preguntas, 4 de ellas abiertas para conocer la opinión de las participantes sobre su experiencia, las 20 restantes con formato tipo Likert de 1 a 5 (muy en desacuerdo a muy de acuerdo), centradas en los siguientes aspectos: a) apoyo emocional (6 preguntas), ejemplo: "en el grupo he tenido un espacio para hablar y reflexionar", b) psicoeducación (5 preguntas), ejemplo: "la información ofrecida por el terapeuta me ha ayudado a comprenderme mejor", c) terapeuta (6 preguntas), ejemplo: "me he sentido motivada por el terapeuta para mejorar" y d) marco terapéutico (4 preguntas), ejemplo: "me hubiera gustado recibir más sesiones". Por último, se les pidió que ordenaran los componentes de la terapia según la utilidad percibida.

\section{Procedimiento}

La primera entrevista de evaluación se realizó de forma individual con aquellos sujetos que accedían voluntariamente al tratamiento y cumplían los criterios de inclusión para formar parte del estudio. En ella se les administraron, previa firma del consentimiento informado, las pruebas psicométricas (tiempo 1 o T1). Después se le citó a una entrevista de devolución de los resultados de las pruebas, en la que, además, se resolvieron dudas acerca del proceso terapéutico y se realizó un contrato terapéutico que tenía en cuenta los horarios y fechas de las sesiones con la finalidad de aumentar la adherencia al tratamiento. En la última sesión del tratamiento se realizó la evaluación post-test (T2), y a los seis meses se realizó de nuevo la evaluación psicométrica (T3). Durante la intervención grupal, las pacientes continuaron asistiendo a las revisiones de psiquiatría, pero se suspendió la intervención psicológica individual. Tras la intervención grupal, aquellas que fueron dadas de alta de psicología, solo continuaron con revisiones en psiquiatría cuando lo requirieron. Aquellas que no fueron dadas de alta, continuaron asistiendo a ambos profesionales.

Se realizaron 12 sesiones semanales y grupales de 1.5 horas con un formato semiestructurado que permitía la participación de las pacientes. Todas las sesiones contaban con la siguiente estructura: a) inicio de la sesión: actividad de saludo, resumen sesión anterior y revisión de tareas para casa; b) desarrollo: abordaje del tema específico a tratar mediante: psicoeducación, ejercicios individuales, grupales, reflexiones, debates, cambio de roles o relajación; y c) cierre: que contemplaba las conclusiones de la sesión, las tareas para casa y una actividad de despedida. La estructura del tratamiento y sus contenidos se detallan en la Tabla 1. Generalmente, 
las sesiones contemplaron elementos de la TCC como: la psicoeducación, entrenamiento en técnicas de asertividad y de habilidades sociales, y elementos de la terapia racional emotiva, combinados con elementos de las terapias de tercera generación, como el trabajo con la compasión, la autocompasión y técnicas de mindfulness. El mensaje de fondo en todas ellas va destinado a la identificación de los roles impuestos socialmente a las mujeres, cómo estos roles influyen en su día a día, y cómo modificar su impacto en ellas. De esta manera, se trabaja con la identificación de las propias necesidades (diferentes a las necesidades de las personas que cuidan o atienden), y con la materialización de estas. Del mismo modo, se entrena a las pacientes en habilidades sociales y en resolución de problemas para que puedan aplicar estas estrategias en su día a día, con el fin de promover el autocuidado y las necesidades propias. Asimismo, se construye una red sólida de apoyo grupal, que se pretende que continúe después del tratamiento. El protocolo detallado se puede conseguir contactando con la autora de correspondencia.

Tabla 1. Esquema de la organización de las sesiones

\begin{tabular}{|c|c|c|c|c|}
\hline Sesión & Objetivos & Contenidos & Técnicas & $\begin{array}{l}\text { Observaciones de } \\
\text { la terapeuta }\end{array}$ \\
\hline $\begin{array}{l}\text { 1. Presentación e } \\
\text { identidad fe- } \\
\text { menina }\end{array}$ & $\begin{array}{l}\text { Conocer a las integrantes del } \\
\text { grupo. } \\
\text { Establecer las normas del } \\
\text { grupo } \\
\text { Descubrir el concepto de roles } \\
\text { de género y su influencia a ni- } \\
\text { vel social y personal. }\end{array}$ & $\begin{array}{l}\text { Presentación de las participantes. } \\
\text { Acuerdo grupal de las normas del } \\
\text { grupo incluida la confidencialidad. } \\
\text { Psicoeducación acerca del gé- } \\
\text { nero, y sus consecuencias sobre } \\
\text { el bienestar psicológico en per- } \\
\text { sonas con identidad de género } \\
\text { femenina. } \\
\text { Acercamiento a la creación de } \\
\text { identidad feminina. }\end{array}$ & $\begin{array}{l}\text { TCC y otros com- } \\
\text { ponentes. }\end{array}$ & $\begin{array}{l}\text { Se observa una tendencia a } \\
\text { locus control externo, una ten- } \\
\text { dencia a la pasividad y a situar- } \\
\text { se en una situación de deses- } \\
\text { peranza. } \\
\text { Se fomenta el proceso activo } \\
\text { de toma de decisiones. }\end{array}$ \\
\hline $\begin{array}{l}\text { 2. Autoestima, } \\
\text { autoconcepto, } \\
\text { autoacepta- } \\
\text { ción y auto- } \\
\text { compasión }\end{array}$ & $\begin{array}{l}\text { Descubrir qué es el autocon- } \\
\text { cepto, la autoestima y cómo } \\
\text { podemos mejorar nuestra } \\
\text { aceptación. } \\
\text { Ahondar en la autoaceptación } \\
\text { y en la autocompasión. } \\
\text { Mejorar la percepción de una } \\
\text { misma. } \\
\text { Aumentar la autocompasión } \\
\text { con una misma. }\end{array}$ & $\begin{array}{l}\text { Psicoeducación y trabajo sobre la } \\
\text { autoestima. } \\
\text { Detección de aspectos que les } \\
\text { hacen sentir valiosas. } \\
\text { Reflexión acerca de los aspectos } \\
\text { que les hacen sentir valiosas y su } \\
\text { relacionan con los roles de género } \\
\text { típicamente femeninos. } \\
\text { Psicoeducación y fomento de la } \\
\text { autoaceptación y autocompasión. }\end{array}$ & $\begin{array}{l}\text { TCC y tercera ge- } \\
\text { neración. }\end{array}$ & $\begin{array}{l}\text { Destacan las cualidades relacio- } \\
\text { nadas con el cuidado del otro } \\
\text { (amable, generosa, paciente y } \\
\text { con capacidad de escucha). } \\
\text { Dificultad para verbalizar cuali- } \\
\text { dades positivas de ellas mismas } \\
\text { "si me quiero soy egoísta" "no } \\
\text { se puede pensar sólo en una } \\
\text { misma" "yo quiero más a los } \\
\text { demás y así me siento útill". } \\
\text { Dificultad del terapeuta para } \\
\text { guiar el grupo de la autocrítica } \\
\text { a la aceptación. }\end{array}$ \\
\hline 3. La queja & $\begin{array}{l}\text { Identificar los motivos de la } \\
\text { conducta de queja y sus sig- } \\
\text { nificados. } \\
\text { Favorecer la comprensión de } \\
\text { los motivos de queja y traduc- } \\
\text { ción en nuevas acciones. }\end{array}$ & $\begin{array}{l}\text { Ejercicios individuales de detec- } \\
\text { ción de pensamientos negativos, } \\
\text { así como emociones y compor- } \\
\text { tamientos asociados a la "queja". } \\
\text { Se comparten con las otras inte- } \\
\text { grantes y se intenta llegar a nue- } \\
\text { vas formas de pensamiento de } \\
\text { forma grupal. } \\
\text { Se realiza un juego de roles con } \\
\text { situacioenes estresantes para las } \\
\text { pacientes donde se practican } \\
\text { nuevas formas de comunicarse } \\
\text { con una misma y con los otros. }\end{array}$ & TCC. & $\begin{array}{l}\text { En esta sesión se observa un } \\
\text { aumento de la cohesión en el } \\
\text { grupo. } \\
\text { Se observan grandes dificul- } \\
\text { tades en el ámbito doméstico } \\
\text { sobre todo en la cooperación } \\
\text { de la realización de las tareas } \\
\text { del hogar. }\end{array}$ \\
\hline
\end{tabular}



4. Habilidades in- Conocer los estilos de reso- terpersonales lución de conflictos. y asertividad
Identificar el estilo habitual propio de resolución de con- flictos.
Desarrollar una forma aserti- va de resolver los conflictos interpersonales.
Aumentar la compasión y au- tocompasión
Psicoeducación de los estilos de resolución de conflictos (pasivo, asertivo y agresivo).
Ejercicio individual de detección del propio estilo.
Entrenamiento en técnicas aser- tivas mediante role-playing por parejas.
Se envía como tarea escribir una carta de agradecimiento.

TCC y tercera generación.

ter

Las pacientes destacan que con anterioridad se sentían más pasivas y ahora se sienten con mayor asertividad.

Aunque refieren un aumento de la tristeza "estoy removida" que se ve asociado a los cambios que han ido realizando día a día en su hogar. Algunas pacientes expresan expectativas positivas sobre la terapia "sé que puedo salir de este estado de ánimo" y otras negativas y de duda "no puedo" "no sé si podré salir de este estado" "no tengo voluntad para cambiar".

5. Red social

Fomentar y aumentar la red de apoyo social recibida y percibida.

TCC.

Todas las pacientes escribieron una carta de agradecimiento a un progenitor fallelidades sociales, el apoyo entre las integrantes del grupo y la calidad de las relaciones.
Se trabaja el desarrollo de habicido.

Se observa intentos de compartir el dolor individual, así como de comparar con el resto las experiencias vividas. Se trabaja la validación del dolor personal, pues se observa una gran capacidad para validar el dolor de otros, pero no el propio.
6. Las crisis vita- Detectar las crisis vitales perles

sonales.
Observar las consecuencias positivas y negativas de estas crisis.

Identificar recursos útiles en crisis anteriores para utilizarlos en el momento presente.
Trabajo individual de detección de situaciones de crisis y de conflicto en la vida personal como momentos de aprendizaje, cambio y crecimiento.

Detección de los aspectos positivos y negativos de las crisis vitales e identificación de recursos para llevar mejor esos momentos.

Puesta en común de habilidades personales para fomentar el empoderamiento femenino y dotar de habilidades a las compañeras.
TCC y otros componentes.
Psicoeducación de explicación de los orígenes de la sintomatología ansiosa, la evidencia de tratamientos actuales.

Ejercicio de relajación.

Realización de práctica de mindfulness que recorre las sensaciones internas y externas.
TCC y tercera generación.
Verbalizan aprendizajes personales gracias a esos momentos difíciles (fortaleza, flexibilidad, autoconocimiento, valorar aspectos positivos, aceptación y disminución de la exigencia).

Comentan situaciones vitales estresantes vividas asociadas socialmente con mayor probabilidad de ocurrencia a las mujeres (cuidado de familiares en situación de dependencia, sufrimiento de agresiones sexuales, situaciones de violencia de género o cargas familiares).

Las participantes refuerzan las habilidades del resto de mujeres para hacer frente a los eventos vitales estresantes.

Comparten sus propios recursos de relajación (lugares seguros, recursos electrónicos y estrategias de disminución del arousal).
Incorporar herramientas para el manejo de la sintomatología ansiosa. 
8. La depresión Potenciar el conocimiento sobre la depresión grupalmente y conocer la experiencia personal.

Adquirir habilidades de manejo de la sintomatología depresiva.
Psicoeducación acerca de: aspectos cognitivos, motores y físicos de la tristeza, la sintomatología depresiva, y los trastornos del estado de ánimo. Más profundamente del TDP. Así como de factores de vulnerabilidad, precipitantes y mantenimiento de esta sintomatología.

Ejercicio de mindfulness de aceptación de las experiencias internas.
TCC y tercera generación.
Verbalizan ideas de debilidad analogía con enfermedad física.

Se observa en la mayoría de las pacientes una intensa lucha interna, autocrítica y culpabilidad por sus síntomas emocionales. Malestar e indefensión por no poder controlar sus emociones y pensamientos.

Culpar al otro "yo quiero es. tar bien pero no me dejan".
9. El autocuidado Conocimiento de la impory las necesi- tancia del autocuidado y de dades propias la autocompasión.

Incorporación de habilidades que potencien el autocuidado y el amor por una misma.
Psicoeducación acerca de la importancia de aprender a cuidarse y ser cuidada y de cómo desarrollar su parte cariñosa, amable y respetuosa hacia ellas mismas.

Psicoeducación acerca del rol femenino impuesto socialmente (atención al cuidado de los otros, sumisión y desatención de las propias necesidades).

Identificación de las propias necesidades.

Psicoeducación de detección de las propias necesidades (comunicar a partir de la necesidad). Ejercicio práctico.

Concepto y práctica de ejercicios de autocompasión (me quiero, me atiendo, soy merecedora de amor).

Se propone que a lo largo de la semana identifiquen una necesidad propia y la manera de atenderla.
TCC y tercera generación.
Dificultad para concretar en acciones "ser feliz, controlar mis pensamientos, no estar deprimida" à "hacer los ejercicios para la espalda, pasear, practicar relajación".

Dificultad para identificar las necesidades asociadas a una misma (y no al cuidado o atención de otras presonas). 10.0bjetivos vitales y actividades gratificantes
Establecer objetivos vitales y actividades gratificantes.

Descubrir o recordar actividades gratificantes de las pacientes.

Darse permiso para el disfrute.
Actividad individual de conocimiento de actividades gratificantes para una misma.

Descubrimiento y asentamiento de objetivos y metas vitales asociados a la satisfacción de necesidades propias.

Planificación, obstáculos y compromiso grupal con las actividades gratificantes personales.
TCC y tercera generación.

Existe cierta dificultad para realizar la tarea enviada el día anterior. Algunas de ellas, señalan necesidades asociadas con el cuidado de otras personas (no el suyo propio). La mayoría de los objetivos están relacionados con su familia. Se hace necesario guiar de lo abstracto a lo específico, y de las necesidades de otros a las propias.

Comparten información sobre recursos de la comunidad. 


\begin{tabular}{|c|c|c|c|c|}
\hline $\begin{array}{l}\text { 11. Pensamientos } \\
\text { negativos }\end{array}$ & $\begin{array}{l}\text { Identificación de pensamien- } \\
\text { tos negativos. } \\
\text { Desarrollo de pensamientos } \\
\text { alternativos. } \\
\text { Introducir la finalización de la } \\
\text { terapia grupal. }\end{array}$ & $\begin{array}{l}\text { Psicoeducación sobre la relación } \\
\text { entre pensamiento-emoción. } \\
\text { Defusión cognitiva. } \\
\text { Mindfulness de los pensamien- } \\
\text { tos y emociones. }\end{array}$ & $\begin{array}{l}\text { TCC y tercera ge- } \\
\text { neración. }\end{array}$ & $\begin{array}{l}\text { Dificultad para diferenciar } \\
\text { emociones y pensamientos. } \\
\text { También para encontrar la } \\
\text { asociación entre los mismos. } \\
\text { Las participantes, logran com- } \\
\text { partir sus emociones (rabia, } \\
\text { tristeza, enfado) con el grupo, } \\
\text { y comentan que les ha resul- } \\
\text { tado muy reparador, ya que } \\
\text { no suelen compartir con otras } \\
\text { personas cómo se sienten. } \\
\text { Muestran expectativas positi- } \\
\text { vas de recuperación. }\end{array}$ \\
\hline $\begin{array}{l}\text { 12. E m p o d e ra - } \\
\text { miento y pre- } \\
\text { vención de } \\
\text { recaídas }\end{array}$ & $\begin{array}{l}\text { Prevenir recaídas. } \\
\text { Cierre y despedida. }\end{array}$ & $\begin{array}{l}\text { Se realiza en primer lugar la } \\
\text { evaluación post-test. } \\
\text { Reflexión sobre el empodera- } \\
\text { miento femenino. } \\
\text { Prevención de recaídas: reflexio- } \\
\text { nar sobre todo lo aprendido en } \\
\text { el grupo como recursos para } \\
\text { afrontar sus dificultades vitales. } \\
\text { Trabajar la despedida del grupo. }\end{array}$ & Cierre. & $\begin{array}{l}\text { Hablan de delegar ciertas ta- } \\
\text { reas, identificar y priorizar sus } \\
\text { propias necesidades, aceptar } \\
\text { la imperfección, del autocui- } \\
\text { dado y de la necesidad de } \\
\text { atenderse. } \\
\text { Intercambian teléfonos crean- } \\
\text { do así una red de apoyo fuera } \\
\text { de la situación de terapia para } \\
\text { el futuro. }\end{array}$ \\
\hline
\end{tabular}

\section{Análisis de datos}

Los datos del estudio fueron analizados con el programa SPSS 26. Calculamos análisis descriptivos, las pruebas de normalidad de Shapiro-Wilk y de homocedasticidad, y como todas las variables en todos los tiempos se distribuyeron de forma normal $(p>.05)$, se procedió a calcular una prueba t de Student para muestras relacionadas. Todo ello, se realizó con la finalidad de analizar las diferencias en los tiempos 1,2 y 3 , y $d$ de Cohen para el cálculo del tamaño del efecto. Según Cohen (1988), se consideran valores pequeños de tamaño del efecto aquellos $\approx 0.2$, medios $\approx 0.5$ y altos $\approx 0.8$. Añadido a lo anterior, se realizaron correlaciones de Pearson entre las variables a estudio.

\section{Resultados}

\section{Estadísticos descriptivos}

Teniendo en cuenta que las puntuaciones iguales o superiores a 9 en el BDI se consideran de presencia de depresión, y que las muestras con psicopatología obtienen medias de aproximadamente 35 puntos en ansiedad estado rasgo, y que las puntuaciones en satisfacción vital pueden llegar hasta 35 puntos, los resultados de la evaluación pretest, señalaron una gran presencia de síntomas ansiosos (en estado y rasgo), depresivos y una baja satisfacción con la vida.

Se observan menores indicadores de psicopatología en el tiempo dos y tres, dándose menores niveles de síntomas ansiosos, depresivos y mayor satisfacción con la vida (Tabla 2).

\section{Diferencias de medias en las variables de estudio en función del momento temporal de evaluación (pre) post-tratamiento y seguimiento).}

Tal y como se mencionó con anterioridad, se comprobó la normalidad de la muestra. Así, en relación con las diferencias estadísticamente significativas entre los diferentes momentos temporales de evaluación (pretest, postest y seguimiento). Encontramos valores mayores de ajuste en las participantes, observándose: menor an- 
siedad (estado y rasgo) y mayor satisfacción con la vida comparándose el T1 con el T2. No obstante, aunque no se dio diferencia estadísticamente significativa en el T1 y el T2 en la sintomatología depresiva, el tamaño del efecto encontrado es moderado. Añadido a lo anterior, pese a no encontrar en otras variables diferencias estadísticamente significativas, observamos generalmente tamaños del efecto o distancias entre las medias moderados-elevados en todos los casos cuando se compararon las medias del T1 con el T2 y del T1 con el T3. Manteniéndose todas las puntuaciones estables entre la evaluación post-tratamiento y el seguimiento, a excepción de la satisfacción con la vida, que disminuyó en el seguimiento (Tabla 2).

Tabla 2. Estadísticos descriptivos del pretratamiento (T1), postratamiento (T2) y seguimiento (T3) y comparaciones de medias

\begin{tabular}{l|cc|cc|ccc|ccc|ccc|cccc}
\hline \multicolumn{3}{|c}{ T1 } & \multicolumn{3}{c}{ T2 } & \multicolumn{3}{c}{ T1-T2 } & \multicolumn{3}{c}{ T3 } & \multicolumn{3}{c}{ T1-T3 } & \multicolumn{3}{c}{ T2-T3 } \\
\hline & M & DT & M & DT & $t$ & $p$ & $d$ & M & DT & $t$ & $p$ & $d$ & $t$ & $p$ & $d$ \\
\hline STAl-E & 46.00 & 10.77 & 31.00 & 10.04 & 5.46 & .003 & 1.44 & 32.00 & 7.00 & 2.14 & .166 & 1.54 & 3.05 & .093 & 0.12 \\
\hline STAI-R & 41.17 & 10.81 & 32.50 & 9.67 & 2.87 & .035 & 0.85 & 32.00 & 4.00 & 2.17 & .162 & 1.13 & 1.00 & .423 & 0.07 \\
\hline BDI & 26.50 & 9.41 & 20.83 & 7.57 & 2.36 & .065 & 0.66 & 22.33 & 11.68 & .57 & .625 & 0.39 & .655 & .580 & 0.01 \\
\hline SWLS & 14.25 & 3.30 & 18.00 & 3.46 & 7.83 & .004 & 1.11 & 11.67 & 5.51 & .862 & .480 & 0.57 & 1.14 & .458 & 1.21 \\
\hline
\end{tabular}

Nota. STAl-E = síntomas ansiosos estado; STAI-R = síntomas ansiosos rasgo; $\mathrm{BDI}=$ síntomas depresivos; $\mathrm{SWLS}=$ satisfacción con la vida; $\mathrm{T1}$ $=$ pretest; $\mathrm{T} 2=$ post-test; $\mathrm{T} 3=$ seguimiento; $M=$ Media; $D T=$ Desviación Típica; $T=t$ de Student; $p=p$ valor; $d=d$ de Cohen

\section{Asociación entre las variables a estudio}

Teniendo en cuenta el bajo tamaño muestral de nuestro estudio piloto, se tomó la decisión de informar de todas las asociaciones lineales moderadas y altas (> .50), fueran o no estadísticamente significativas (Tabla 4). Los pacientes que tenían mayor sintomatología ansiosa mostraron también mayor sintomatología depresiva y menos satisfacción con la vida.

En relación con las asociaciones encontradas, observamos a grandes rasgos, que aquellas pacientes con más sintomatología ansiosa (estado y rasgo) también presentaron más sintomatología depresiva y menor satisfacción vital en todos los tiempos de evaluación, excepción del seguimiento donde la variable satisfacción con la vida solo se asoció de forma moderada con la ansiedad como rasgo (Tabla 3).

\section{Mejoría clínica y subjetiva de las pacientes}

Al finalizar el tratamiento grupal se pactó el alta con el 50\% de las pacientes por mejoría (puntuaciones menores o iguales a 9 en BDI) mientras que las otras se reincorporaron a sus tratamientos psicológicos individuales. Además de lo anterior, valoraron mediante la encuesta creada ad hoc, su experiencia con el tratamiento $(M=3.80 ; D T=0.44)$, los cambios conductuales percibidos $(M=4.00 ; D T=0.00)$ y el alivio de síntomas emocionales $(M=3.60 ; D T=0.54)$ como moderados o elevados, observándose una moderada o elevada sensación de mejoría subjetiva.

\section{Satisfacción con el tratamiento}

Las pacientes valoraron mediante la encuesta de satisfacción, como especialmente positivo el apoyo social percibido por las compañeras y la terapeuta $(M=4.34 ; D T=0.32)$, la información recibida $(M=4.40 ; D T=$ $0.43)$, y la relación con el profesional que ha impartido las sesiones $(M=4.85 ; D T=0.14)$. Valoran como moderadamente satisfactorio el marco o encuadre de la terapia $(M=2.25 ; D T=0.25)$, señalando en las opciones 
Tabla 3. Asociación entre las variables a estudio

\begin{tabular}{|c|c|c|c|c|c|c|c|c|c|c|c|c|c|}
\hline & & \multicolumn{4}{|c|}{$\mathrm{T} 1$} & \multicolumn{4}{|c|}{ T2 } & \multicolumn{4}{|c|}{ T3 } \\
\hline & & STAl-E & STAI-R & BDI & SWLS & STAl-E & STAl-R & BDI & SWLS & STAI-E & STAI-R & BDI & SWLS \\
\hline \multirow{4}{*}{$\digamma$} & STAl-E & 1 & & & & & & & & & & & \\
\hline & STAI-R & .745 & 1 & & & & & & & & & & \\
\hline & BDI & $.921^{\star \star}$ & $.920^{\star \star}$ & 1 & & & & & & & & & \\
\hline & SWLS & $-.947^{\star \star}$ & -.796 & $-.966^{\star \star}$ & 1 & & & & & & & & \\
\hline \multirow{4}{*}{$\cong$} & STAI-E & .793 & $.831^{\star}$ & .806 & -.878 & 1 & & & & & & & \\
\hline & STAI-R & .701 & .745 & .704 & -.739 & $.831^{*}$ & 1 & & & & & & \\
\hline & BDI & .782 & .699 &, 781 & -.828 & $.970^{* \star}$ & $.845^{*}$ & 1 & & & & & \\
\hline & SWLS & -.824 & $-.903^{\star}$ & $-.908^{\star *}$ & $-.961^{* \star}$ & $-.979^{* *}$ & $-.908^{*}$ & $-.891^{*}$ & 1 & & & & \\
\hline \multirow{4}{*}{$\stackrel{m}{p}$} & STAl-E & .957 & .969 & $.990^{*}$ & $-.990^{\star}$ & .979 & .979 & $.999^{*}$ & $-1.00^{\star \star}$ & 1 & & & \\
\hline & STAI-R & .931 & .915 & .866 & -.866 & $.982^{*}$ & .655 & .807 & $-1.00^{\star \star}$ & .982 & 1 & & \\
\hline & BDI & .980 & .971 & .939 & -.939 & .958 & .776 & .897 & $-1.00^{* *}$ & .881 & .985 & 1 & \\
\hline & SWLS & I & 1 & I & 1 & 1 & I & I & 1 & 1 & .545 & 1 & 1 \\
\hline
\end{tabular}

Nota. STAl-E = síntomas ansiosos estado; STAI-R = síntomas ansiosos rasgo; $\mathrm{BDI}$ = síntomas depresivos; SWLS = satisfacción con la vida; T1 $=$ pretest; $\mathrm{T} 2=$ post-test; $\mathrm{T} 3=$ seguimiento; $M=$ Media; $D T=$ Desviación Típica; ${ }^{*} p \leq .05 ;{ }^{* *} p \leq .01$

de respuesta abiertas que el lugar de realización no era suficientemente silencioso, pues se realizó en la unidad de salud mental, donde puede haber distracciones. Además, señalaron la necesidad de aumentar el número de sesiones de terapia para consolidar más aún si cabe los aprendizajes realizados. Añadido a lo anterior, solicitaron más técnicas de relajación, pues fue una herramienta que aplicaron en su día a día y que señalaron que les resultó eficaz para relajarse y sentirse en paz con ellas mismas.

Como aspecto positivo, remarcaron la posibilidad de encontrar un lugar seguro donde poder compartir su experiencia, conociendo personas que estaban pasando por su misma situación, y la posibilidad que les ha brindado el tratamiento de valorarse más, identificar las conductas que estaban asociadas al rol de género femenino e intentar paliarlas. Por otra parte, valoraron la estructura y características del programa como muy positivas en general, destacando que se habían sentido comprendidas y valoradas. Por último, las pacientes consideraron que lo más útil del tratamiento para ellas fue el apoyo grupal (47.83\%), seguido del apoyo por parte del terapeuta $(21.74 \%)$, de la información transmitida por parte del terapeuta $(8.70 \%)$, de las técnicas de relajación $(8.70 \%)$ y de las tareas enviadas para realizar en casa $(4.35 \%)$.

\section{Discusión y conclusiones}

Con relación al objetivo planteado, desarrollamos un programa de intervención multicomponente para mujeres con TDP, que integró aspectos cognitivo-conductuales, técnicas basadas en la autocompasión y la atención plena y la perspectiva de género. El programa se implementó en una muestra piloto de 6 mujeres que participaron voluntariamente y obtuvo resultados muy satisfactorios.

En respuesta a la $\mathrm{H} 1$, observamos que las pacientes tras la intervención muestran menos sintomatología ansiosa, no solo en estado, sino también como rasgo. Además, informan de menos síntomas depresivos. Aunque 
en el resto de las variables no encontramos diferencias estadísticamente significativas, se dieron tamaños del efecto desde moderados a muy elevados en todas las comparaciones. Estos valores son superiores a los encontrados en otros trabajos realizados en trastornos emocionales (Cruijpers, van Straten, Oppen y Anderson, 2008; Pinquart, Dubertein y Lyness, 2006; Rosenbaum, Tiedemann, Sherrington, Curtis y Ward, 2014; Sanz-Cruces et al., 2018), si bien, hasta donde conocemos, todavía no disponemos de trabajos específicos realizados en personas con TDP.

Así, se observa además de los cambios en sintomatología ansiosa y depresiva, una tendencia a la mejoría de la satisfacción vital. Aspectos que se mantienen seis meses después de la intervención, a excepción de la satisfacción con la vida. Nuestros datos vienen a confirmar los de estudios anteriores donde mediante intervenciones grupales multicomponente se muestran útiles para la reducción de la sintomatología ansiosa y depresiva y el aumento de la satisfacción vital (Coto-Lesmes et al., 2020; García et al., 2020; Hamidian et al., 2013; Rockliff et al., 2011; Otero et al., 2015; Sanz et al., 2003).

Por otra parte, en relación con las asociaciones encontradas en nuestra muestra, aquellas pacientes que muestran mayor sintomatología ansiosa (estado y rasgo), también obtienen mayores indicadores de depresión y una menor satisfacción con la vida, tal y como indicaban estudios previos (Acosta, 2019; Teixeira y Prebianchi, 2019).

Tras nuestros resultados y basándonos en la evidencia previa, podemos observar cómo en nuestra muestra de mujeres con TPD, la sintomatología emocional (ansiosa y depresiva) así como la satisfacción vital, puede mejorar tras una intervención cognitivo conductual, con elementos coadyuvantes (técnicas basadas en la autocompasión y mindfulness) y una perspectiva de género, necesaria pero poco utilizada (Ebrahimi et al., 2013; Feldman, 2007; Guhn et al., 2019; Hamidian et al., 2013; Ministerio de Sanidad, Servicios Sociales e Igualdad, 2014; Muñoz et al., 2008; Narbona et al., 1993; Pérez y García, 2001). Así, las participantes han aprendido a identificar los roles de género impuestos socialmente, así como su influencia en su día a día, y han encontrado recursos apoyados en la TCC, la terapia basada en la autocompasión y la MBCT para hacerles frentes, atendiendo a sus necesidades, fomentando el autocuidado y aprendiendo nuevas técnicas de relacionarse con otras personas y relajarse, potenciando así sus recursos personales.

A pesar de las contribuciones señaladas anteriormente, los resultados obtenidos solo pueden considerarse preliminares debido a las limitaciones del presente estudio. Las características de nuestra muestra, la perdida de muestra en el seguimiento, así como el tipo de muestreo realizado, complican la generalización de los resultados a la población general. Ya que la selección de la muestra es incidental, lo que puede afectar a la validez de los resultados, que pueden deberse a variables no controladas como los cambios en la motivación de los pacientes a lo largo del tratamiento o su disponibilidad. Los evaluadores, la terapeuta y las pacientes no son ciegos, y lo más importante, no contamos con un grupo control, lo que nos permitiría asociar con mayor evidencia los cambios a la intervención. Además, no se evaluó si se dieron altas en el servicio de psiquiatría tras la intervención, por lo que futuras intervenciones, deberían de contemplar este aspecto.

Futuras líneas de investigación, deberían aumentar la muestra de estudio e idear formas de disminuir la pérdida de la muestra en el seguimiento (recordatorios, o uso de las tecnologías de la comunicación e información por ejemplo) (Molés et al., 2015), así como evaluar otras variables que puedan estar interfiriendo en el ajuste psicológico de las pacientes, como: el apoyo social percibido, las habilidades sociales, el padecimiento de situaciones vitales estresantes, el cuidado de personas dependientes o la motivación hacia el tratamiento psicológico (Cortés et al., 2020; Guhn et al., 2019; Montesó-Curto, 2014; Pla et al., 2013; Saenz-Miguel et al., 2019; Torres et al., 2019), aumentando así la validez del estudio. No obstante, podemos destacar que se trata de un estudio piloto realizado en un ambiente clínico real, lo que puede aumentar la validez ecológica del estudio. Añadido a lo anterior, se realizó en un espacio familiar para el paciente, lo que reduce los sesgos de reactividad ante la situación de evaluación mejorando así la validez. De esta manera, observamos que la media de edad de nuestras pacientes es mucho más elevada que la de otros estudios (Berrio et al., 2019; De Francisco y Blasco, 2015; Ebrahimi et al., 2013), y por tanto más representativa de la realidad clínica. Además de lo anterior, contamos con la perspectiva cuantitativa pero también cualitativa, que hace enriquecedor nuestro estudio. Las pacientes con TDP, no solo puntúan significativamente menos en ansiedad y depresión y más en satisfacción vital, sino que afirman sentirse muy satisfechas con los diversos aspectos de la terapia, percibiendo 
una mejoría moderada-elevada en su experiencia subjetiva y alivio de síntomas y en relación con los cambios conductuales, pero solicitan que, se realicen más sesiones, con mayor frecuencia y duración. Añadido a lo anterior, el 50\% de las participantes recibió el alta de la consulta de psicología, lo que supone una mejor gestión de los recursos personales, económicos e institucionales (Sanz-Cruces et al., 2018). Estos datos van en la línea de otros estudios realizados con terapia cognitivo conductual o multicomponentes en personas con otros trastornos emocionales, que encontraban altas alrededor del 50\% de los casos (Poves et al., 2010; Sanz et al., 2014).

Cabe señalar que, pese a la estabilidad de las puntuaciones en la sintomatología ansiosa y depresiva, la satisfacción vital disminuye notablemente a los seis meses de finalizar la terapia. Pensamos, que, si la red de apoyo social generada en la terapia se mantuviera a lo largo del tiempo, estas pacientes, podrían sentirse más satisfechas (Acosta, 2019).

El formato grupal mejora la eficiencia del trabajo, ya que permite llegar a un mayor número de pacientes con menores recursos (temporales, espaciales y personales), lo que reduce el tiempo de listas de espera en las consultas de las USM y el tiempo entre sesiones. Añadido a lo anterior, la terapia grupal favorece la identificación y el apoyo entre pares, generando una red social de apoyo y un espacio de intercambio y aprendizaje. Al mismo tiempo, fomenta: la toma de conciencia, la actitud activa en la producción de su salud, el autoconocimiento y la identificación de las propias necesidades, y la expresión de los problemas y la búsqueda de soluciones alternativas, disminuyendo las resistencias al cambio, estimulando el crecimiento personal, disminuyendo así la sintomatología ansiosa y depresiva y aumentando la satisfacción vital (Acosta, 2019; Latorre et al., 2012; Sanz-Cruces et al., 2016; Sanz-Cruces et al., 2018; Teixeira y Prebianchi, 2019; Vinogradov y Yalom, 1996).

A la luz de los resultados obtenidos, podemos concluir que nuestros datos muestran que nuestra intervención multicomponente con enfoque de género es una herramienta útil para: disminuir la sintomatología ansiosa y depresiva y aumentar la satisfacción vital en mujeres con TDP. Lo anterior, puede tener una gran repercusión en la salud mental y física de las participantes (Rockliff et al., 2011). Debemos entender nuestra investigación como una experiencia con gran valor debido a la utilidad en nuestro contexto, que puede sentar las bases para iniciar y mejorar programas enfocados a promover la salud de nuestra población a través de intervenciones psicológicas eficaces, enfatizando al mismo tiempo un uso eficiente de los recursos humanos y económicos.

\section{Referencias}

Acosta, L. D. (2019). Factores asociados a la satisfacción vital en una muestra representativa de personas mayores de Argentina. Hacia Promoción de Salud, 24(1), 56-69. https://doi.org/10.17151/hpsal.2019.24.1.6 Alonso-Llácer, L. (2017). Mindfulness y duelo: cómo la serenidad mindful y la compasión contribuyen al bienestar tras la pérdida (Tesis doctoral). Universidad de Valencia, España. http://hdl.handle.net/10550/59253

Angst, J., Gamma, A., Rossler, W., Ajdacic, V. y Klein, D. N. (2009). Long-term depression versus episodic major depression: results from the prospective Zurich study of a community sample. Journal of Affective Disorders, 115(1-2), 112-121. https://doi.org/10.1016/j.jad.2008.09.023

Asociación Americana de Psiquiatría (2014). Manual diagnóstico y estadístico de los trastornos mentales (5 ed.). Panamericana.

Atienza, F., Pons, D., Balaguer, I. y García-Merita, M. (2000). Propiedades psicométricas de la escala de satisfacción con la vida en adolescentes. Psicothema, 12(2), 314-319.

Barnhofer, T. (2019). Mindfulness training in the treatment of persistent depression: Can it help to reverse maladaptive plasticity? Current opinion in psychology,28,262-267. https://doi.org/10.1016/j.copsyc.2019.02.007

Beck, A. T. y Steer, R. A. (1987). Manual for the Revised Beck Depression Inventory. Psychological Corporation.

Berrío, N. (2019). Terapia cognitiva con entrega virtual en trastorno depresivo persistente: estudio de caso. Revista Clínica Contemporánea, 10 Artículo e21, 1-14. https://doi.org/10.5093/cc2019a21

Cerdá, J., Benito, A., Mejías, A., Bonora, Y., Bertó, R. y Pretel, J. (2017). Eficacia del grupo de relajación en una unidad de salud mental: estudio piloto. Revista de enfermería y salud mental, 8, 15-24. 
Cohen, J. (1988). Statistical power analysis for the behavioral sciencies (2nd ed.). Erlbaum.

Cortés, L., Lacomba-Trejo, L. y Pérez-Marín, M. (2020). Levantando la voz: las cuidadoras en el hogar de personas en situación de dependencia. Trabajo Social Hoy, 89, 83-100. https://doi.org/10.12960/TSH.2020.0006

Coto-Lesmes, R., Fernández-Rodríguez, C. y González-Fernández, S. (2020). Activación Conductual en formato grupal para ansiedad y depresión. Una revisión sistemática. Terapia Psicológica, 38(1), 63-84.

Cuijpers, P., Andersson, G., Donker, T. y van Straten, A. (2011). Psychological treatment of depression: results of a series of meta-analyses. Nordic Journal of Psychiatry, 65(6), 354-364. https://doi.org/10.3109/080394 88.2011 .596570

Cuijpers, P., van Straten, A., van Oppen, P. y Anderson, G. (2011). Are psychological and pharmacologic interventions equally effective in the treatment of adult depressive disorders? A meta-analysis of comparative studies. 2008. In: Database of Abstracts of Reviews of Effects (DARE): Quality-assessed Reviews. Centre for Reviews and Dissemination (UK). https://www.ncbi.nlm.nih.gov/books/NBK98252/

de Francisco, C. C. y Blasco, R. S. (2015). Intervención cognitivo conductual en un caso de distimia con componentes de ansiedad de evaluación: Análisis de un caso clínico. Revista de Casos Clínicos en Salud Mental, 3(1), 43-59.

Diener, E., Emmons, R., Larsen, R. J. y Griffin, S. (1985). The Satisfaction with Life Scale. Journal of Personality Assessment, 49(1), 71-75. https://doi.org/10.1207/s15327752jpa4901 13

Ebrahimi, A., Neshatdoost, H. T., Mousavi, S. G., Asadollahi, G. A. y Nasiri, H. (2013). Controlled randomized clinical trial of spirituality integrated psychotherapy, cognitive-behavioral therapy and medication intervention on depressive symptoms and dysfunctional attitudes in patients with dysthymic disorder. Advanced Biomedical Research, 2(2), Artículo 53. https://doi.org/10.4103/2277-9175.114201

Feldman, G. (2007). Cognitive and behavioral therapies for depression: overview, new directions, and practical recommendations for dissemination. Psychiatric Clinics of North America, 30(1), 39-50. https://doi. org/10.1016/i.psc.2006.12.001

García, L., Quintana-Orts, C. y Rey, L. (2020). Cibervictimización y satisfacción vital en adolescentes: la inteligencia emocional como variable mediadora. Revista de Psicología Clínica con Niños y Adolescentes, 7(1), 38-45. https://doi.org/10.21134/rpcna.2020.07.1.5

Gallardo-Moreno, G., Jiménez-Maldonado, M., González-Garrido, A. y Villaseñor-Cabrera, T. (2013). La distimia como entidad nosológica. Algunas consideraciones clínicas, epidemiológicas, etiológicas y de utilidad diagnóstica. Revista Mexicana Neurociencia, 14( 4), 215-222.

Guhn, A., Steinacher, B., Merkl, A., Sterzer, P. y Köhler, S. (2019). Negative mood induction: Affective reactivity in recurrent, but not persistent depression. PLOS ONE, 14(1). https://doi.org/10.1371/journal. pone.0208616

Gureje, O. (2011). Dysthymia in a cross-cultural perspective. Current opinion in psychiatry, 24(1), 67-71. https://doi.org/10.1097/YCO.0b013e32834136a5

Hamidian, S., Omidi, A., Mousavinasab, S. M. y Naziri, G. (2013). Comparison of the effect of mindfulness-based cognitive therapy accompanied by pharmacotherapy with pharmacotherapy alone in treating dysthymic patients. Iranian Red Crescent Medical Journal, 15(3), 239-244. https://doi.org/10.5812/ircmj.8024

Institute for Health Metrics and Evaluation. (2017). Global Burden of Disease Study 2017. http://ghdx. healthdata.org/gbd-2017

Irastorza Eguskiza, L. J. (2012). Personalidad depresiva: Concepto y diferenciación: diagnóstico diferencial entre trastorno de personalidad depresiva y distimia [Tesis doctoral no publicada]. Universidad Complutense de Madrid, España.

Korman, G. P. y Garay, C. J. (2012). El modelo de terapia cognitiva basada en la conciencia plena (mindfulness). Revista Argentina de Clínica Psicológica, 21(1), 5-14.

Latorre, J. M., Navarro, B., Parra, M., Salguero, J. M., Wood, C. M. y Cano, A. (2012). Evaluación e intervención de los problemas de ansiedad y depresión en atención primaria: un problema sin resolver. Revista Clínica de Medicina de Familia, 5(1), 37-45.

Ministerio de Salud. (2013). Guía Clínica Depresión en personas de 15 años y más. Minsal Santiago. 
Ministerio de Sanidad y Consumo. (2007). Estrategia en salud mental del SNS 2006. https://www.mscbs.gob. es/organizacion/sns/planCalidadSNS/pdf/excelencia/salud mental/ESTRATEGIA SALUD MENTAL SNS PAG WEB.pdf

Ministerio de Sanidad, Servicios Sociales e Igualdad. (2014). Guía de práctica clínica sobre el manejo de la depresión en el adulto. https://portal.guiasalud.es/wp-content/uploads/2018/12/GPC 534 Depresion Adulto Avaliat compl.pdf

Molés, M., Quero, S., Pérez, M.A., Nebot, S. y Botella, C. (2015). Eficacia diferencial de dos formas de aplicar las tareas para casa en el tratamiento de los trastornos adaptativos. Terapia emocional on line vs. formato tradicional. Ágora de Salut, 1, 245-257. https://doi.org/10.6035/AgoraSalut.2015.1.19

Montesó-Curto, P. (2014). La construcción de los roles de género y su relación con el estrés crónico y la depresión en las mujeres. Comunitania. Revista Internacional de Trabajo Social y Ciencias Sociales, 8, 105-126. https://doi.org/10.5944/comunitania.8.6

Moscoso, M. S. (2019). Mindfulness, autorregulación emocional y reducción de estrés: ¿ciencia o ficción? Revista Mexicana de Investigación en Psicología, 10(2), 101-121.

Muñoz, M. A., De Casas, M. X., Cobo, R., Fontcuberta, J., Manrique, R. y Pérez, F. (2008). Malestar psicosocial de la mujer: Experiencia de una intervención grupal en atención primaria. Archivos en Medicina Familiar, 10(3), 96-100.

Narbona, G., Gómez, M. I. y Martinez, B. (1993). Terapia grupal cognitiva en distimia. Maristan, 11, 60-63.

Nobile, M., Cataldo, G. M., Marino, C. y Molteni, M. (2003). Diagnosis and treatment of dysthymia in children and adolescents. CNS drugs, 17(13), 927-946. https://doi.org/10.2165/00023210-200317130-00001

OMS. (2017). Depression and Other Common Mental Disorders: Global Health Estimates.

OMS. (2018). Depresión. https://www.who.int/es/news-room/fact-sheets/detail/depression

Ortuño-Sierra, J., García-Velasco, L., Inchausti, F., Debbané, M. y Fonseca-Pedrero, E. (2016). Nuevas aproximaciones en el estudio de las propiedades psicométricas del STAI. Actas Españolas de Psiquiatría, 44(3), 83-92.

Otero, J., Ales, A. y Vucinovich, N. (2015). Grupos de alta para pacientes con distimia crónica: Una propuesta psicoterapéutica orientada al fin de tratamiento. Revista de la Asociación Española de Neuropsiquiatría, 35(127), 527-540. https://doi.org/10.4321/S0211-57352015000300006

Pérez, M. y García, J. M. (2001). Tratamientos psicológicos eficaces para la depresión. Psicothema, 13(3), 493-510.

Pla, I., Adam, A. y Bernabeu, I. (2013). Estereotipos y prejuicios de género: factores determinantes en salud mental. Norte de Salud Mental, 11(46), 20-28.

Pinquart, M., Duberstein, P. R. y Lyness, J. M. (2007). Effects of psychotherapy and other behavioral interventions on clinically depressed older adults: A meta-analysis, Aging \& Mental Health, 11(6), 645-657, https:// doi.org/10.1080/13607860701529635

Poves, S., Romero, R., y Vucínovich, N. (2010). Experiencia grupal breve para pacientes con trastornos de ansiedad en un Centro de Salud Mental. Asociación Española de Neuropsiquiatría, 30(3), 409-418. https:// doi.org/10.4321/S0211-57352010000300004

Rockliff, H., Karl, A., Mcewan, K., Gilbert, J., Matos, M. y Gilbert, P. (2011). Effects of Intranasal Oxytocin on 'Compassion Focused Imagery'. Emotion, 11(6), 1388-1396. https://doi.org/10.1037/a0023861

Rosenbaum, S., Tiedemann, A., Sherrington, C., Curtis, J. y Ward, P. (2014). Physical Activity Interventions for people with mental illness: A systematic review and meta-analysis. The Journal of Clinical Psychiatry, 75(9), 964-974. https://doi.org/10.4088/JCP.13r08765

Rossel, L., Erazo, R., Nieto, R., Fuentes, C. y Curivil, P. (2020). Uso de criterios y guías clínicas en la creación de una unidad de trastornos del ánimo. Revista Médica Clínica Las Condes, 31(2), 114-121. https://doi. org/10.1016/j.rmclc.2020.02.003

Saenz-Miguel, S., Runzer-Colmenares, F. M. y Parodi, J. F. (2019). Trastornos depresivos en adultos mayores de once comunidades altoandinas del Perú 2013-2017. Acta Médica Peruana, 36(1), 26-31. https://doi. org/10.35663/amp.2019.361.632 
Sanz, J. M., García, I. M. y Carbajo, E. (2014). Tratamiento del Duelo en Salud Mental: Una Experiencia Grupal. Revista de Psicoterapia, 25(99), 115-133. https://doi.org/10.33898/rdp.v25i99.22

Sanz-Cruces, J. M., García-Cuenca, I. M., Cuquerella, M. A., Cano, I., Jordá, E., Blasco J. y Carbajo E. (2016). Intervención Grupal Cognitivo Conductual en el Trastorno Adaptativo. Behavioral Psychology, 24(3), 389403.

Sanz-Cruces, J. M., García-Cuenca, I. M., Lacomba-Trejo, L., Cuquerella, M. A., Cano, I., Ferrandis, M., Jordá,E. y Carbajo, E. (2018). Group therapy for patients with adjustment disorder in primary care. The Spanish Journal of Psychology, 21 Artículo e50. https://doi.org/10.1017/sjp.2018.51

Sanz, J., Perdigón, A. L. y Vazquez, C. (2003). Adaptación española del Inventario para la Depresión de BeckII (BDI-II): 2. Propiedades psicométricas en población general. Clínica y Salud, 14(3), 249-280.

Spielberger, C. D., Gorsuch, R. L. y Lushene, R. (1982). Manual del Cuestionario de Ansiedad Estado/Rasgo (STAI). TEA Ediciones.

Steer, R. A. y Beck, A. T. (1988). Beck Depression Inventory. En M. Hersen y A. S. Bellack (Eds.), Dictionary of behavioral assessment techniques. Pergamon Press.

Teixeira, F. D. y Prebianchi, H. B. (2019). Comprometimento, estresse e satisfação com a vida de profissionais da saúde. Revista Psicologia: Organizações e Trabalho, 19(2), 598-606. https://doi.org/10.17652/ rpot/2019.2.15321

Torres, A., Ortiz, Y., Martínez, H., Fernández, Y. N. y Pacheco, B. (2019). Factors associated with adherence to treatment in patients with depression. Multimed. Revista Médica, 23(1), 1-10.

Uher, R. (2014). Persistent depressive disorder, dysthymia, and chronic depression: Update on diagnosis, treatment. Psychiatric Times, 31(8), 46-46.

Vallejo, M. Á. (2006). Mindfulness. Papeles del psicólogo, 27(2), 92-99.

Vázquez, C., Duque, A. y Hervás, G. (2013). The Satisfaction with Life Scale (SWLS): Adaptation and normative data in a spanish representative national sample. Spanish Journal of Psychology, 16, Artículo E82. https://doi.org/10.1017/sjp.2013.82

Vinogradov, S. y Yalom, I. (1996). Guía breve de psicoterapia de grupo. Paidós.

Artículo recibido: $27 / 05 / 2020$

Artículo aceptado: 12/01/2021 